\title{
Laboratory investigation of platelet function: a review of methodology
}

\author{
DA YARDUMIAN, IJ MACKIE, SJ MACHIN \\ From the Department of Haematology, Middlesex Hospital, London
}

SUMMARY Over the past decade interest in and knowledge about the role of platelets in the haemostatic process and in various pathological conditions has continued to grow. The scope of laboratory methodology to investigate platelet function in clinical haemorrhagic and thrombotic disorders in the specialised haemostasis unit has also proportionally widened. After highlighting the physiological processes of the role of platelets in the haemostatic mechanism this brief review comments critically on the available routine techniques used to study platelet function in patients who present primarily with a bleeding tendency.

\section{Physiology of platelet function in haemostasis}

Haemostatic reactions can be classified into several overlapping and sequential events: localised vasoconstriction at the site of vessel injury: platelet adhesion to exposed subendothelial basement membrane and collagen fibres; formation of a platelet aggregate or plug; activation of the coagulation cascade leading to formation of fibrin, which reinforces the platelet plug; and finally, activation of the fibrinolytic system, which digests the haemostatic plug and allows growth of new vascular endothelial cells to complete the repair process. ${ }^{1}$

Platelets circulate as non-nucleated discs and consist of a trilaminar phospholipoprotein membrane with submembrane circumferential contractile filaments, three types of granules, and an irregular internal network of canaliculi, through which the granule contents can be released on to the platelet surface. $^{2}$

The granule types are: dense granules which release adenosine diphosphate (ADP), adenosine triphosphate (ATP), serotonin, and calcium ions; $\alpha$ granules whose release constituents include platelet derived growth factor, platelet factor 4 with heparin neutralising ability, $\beta$ thromboglobulin, factor VIII related antigen/von Willebrand factor; factor $\mathrm{V}$, fibrinogen, fibronectin, and probably thrombospondin; and lysosomal granules.

Table 1 summarises the subsequent interactions with circulating platelets, which occur after damage to the vessel wall. When the vessel wall is damaged

Accepted for publication 3 March 1986 subendothelial structures, including basement membrane collagen and microfibrils, are exposed. Circulating platelets react with exposed collagen fibres, and their adherence to the damaged surface is mediated by high molecular weight multimers of the factor VIII von Willebrand factor and probably fibronectin. Details of the structure and physiological importance of von Willebrand factor have been reviewed extensively elsewhere. ${ }^{3}$

When activated, platelets immediately change shape, losing their discoid shape, and form tiny spheres with numerous projecting pseudopods. After adhesion of a single layer of platelets to the damaged vascular endothelium platelets stick to one another and form aggregates. Certain substances react with specific platelet membrane surface receptors and ini-

Table 1 Platelet function in haemostatic process

Damaged vessel wall
Platelet adhesion and plasma VIIIvWf *multimers interaction
Activation of platelet membrane receptors
Platelet shape change and aggregation
Platelet activation proceeds by:
arachidonic acid metabolism;
increase of free cytoplasmic calcium ions;
release of platelet activating factor
Release of granule contents
$\downarrow$
Irreversible aggregate formed
$\downarrow$

*factor VIII/von Willebrand factor 


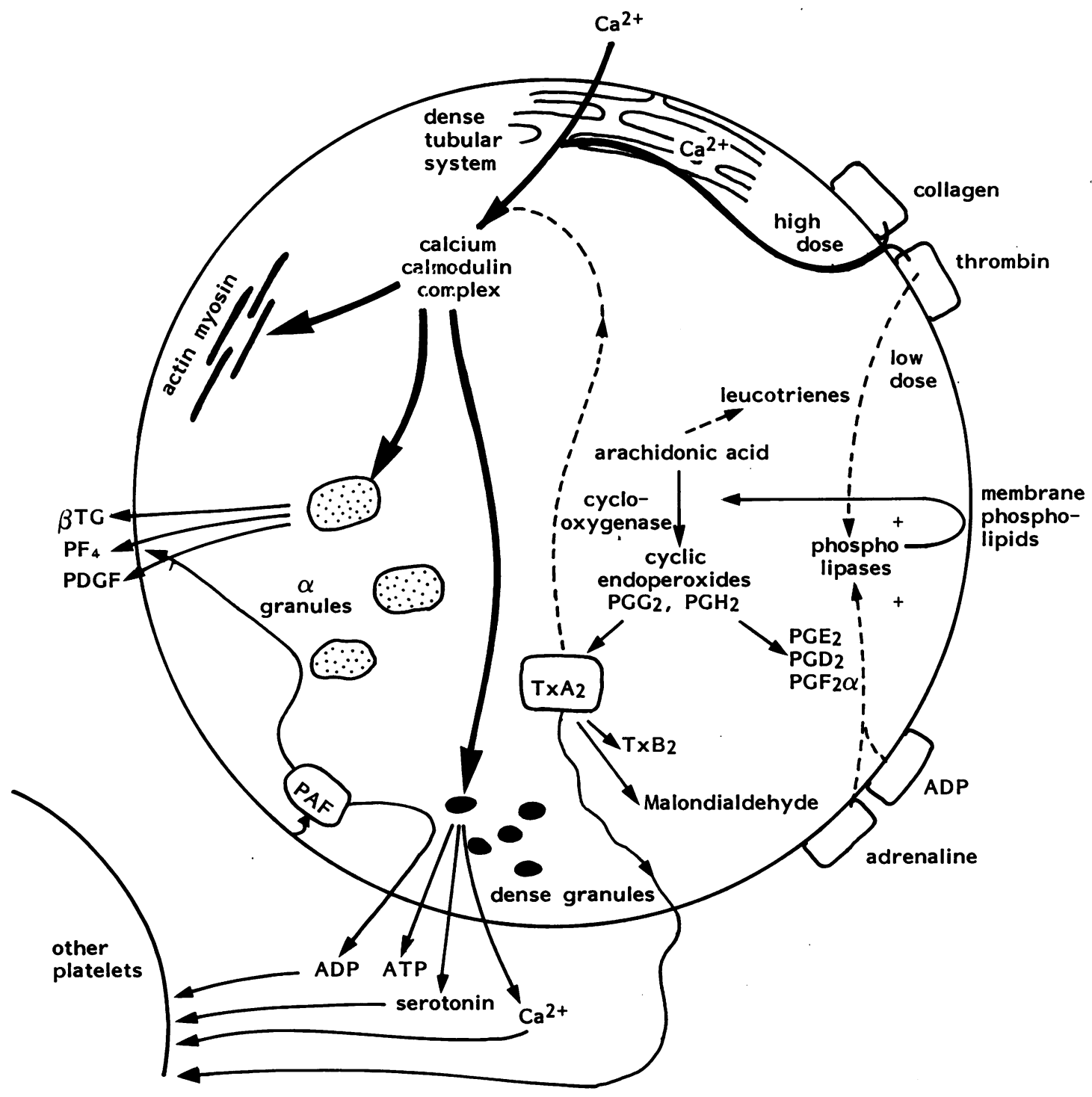

Fig. 1 Schematic representation of pathways of platelet activation. $\mathrm{Ca}^{++}=$calcium ions; $P G G_{2}, \mathrm{H}_{2}, E_{2}, \mathrm{D}_{2}$, and $\mathrm{F}_{2 \alpha}=$ prostaglandins $G_{2}, H_{2}, E_{2}, D_{2}$, and $F_{2 \alpha}$, respectively. $T X A_{2}=$ thromboxane $A_{2} ; T X B_{2}=$ thromboxane $B_{2}$;

$\beta-T G=\beta$ thromboglobulin; $P F_{4}=$ platelet factor $4 ; P D G F=$ platelet derived growth factor; $A D P=$ adenosine diphosphate; $A T P=$ adenosine triphosphate.

tiate platelet aggregation and further activation. These include exposed collagen fibres, ADP, adrenaline, serotonin, thrombin, and certain arachidonic acid metabolites, including thromboxane $\mathrm{IA}_{2}\left(\mathrm{TXA}_{2}\right)$. In areas of non-linear blood flow, such as that which occurs at the site of an injured vessel wall or atherosclerotic plaque, locally damaged red cells release ADP which further activates platelets. ${ }^{4}$
Human platelet aggregation may occur by at least two and possibly three independent but related path-? ways (Fig. 1). The first pathway of activation entails 0 arachidonic acid metabolism. ${ }^{5}$ Activation of phospholipase enzymes releases free arachidonic acid from $\stackrel{\mathbb{D}}{\Phi}$ the membrane phospholipids. Roughly $50 \%$ of free $\stackrel{\mathbb{\Omega}}{\Omega}$ arachidonic acid is converted by a lipoxygenase $\overline{-}$ enzyme to a series of products including various 
leucotrienes, which probably have only a small role in the control of haemostasis, being more important as chemoattractants of white cells. ${ }^{6}$ The remaining arachidonic acid is converted by the enzyme cyclooxygenase into the cyclic endoperoxides $\mathrm{PGG}_{2}$ and $\mathrm{PGH}_{2}$, which are very labile. Most of the endoperoxides are then rapidly converted by the thromboxane synthetase enzyme complex into $\mathrm{TXA}_{2}$. $\mathrm{TXA}_{2}$ has profound biological activity causing platelet granule release and local vasoconstriction, and after release stimulates other platelets to aggregate locally. It exerts these effects by raising intracytoplasmic free calcium ion concentrations and binding to specific granule receptors. TXA $\mathrm{T}_{2}$ is a very labile product with a half life in vivo of about $45 \mathrm{sec}-$ onds before it is degraded into the inactive compounds thromboxane $\mathrm{B}_{2} \quad\left(\mathrm{TXB}_{2}\right)$ and malondialdehyde. A small proportion of the cyclic endoperoxides are converted to the primary prostaglandins $\mathrm{PGE}_{2}, \mathrm{PGD}_{2}$, and $\mathrm{PGF}_{2}$.

The second pathway of platelet activation can proceed completely independently of arachidonic acid metabolism and thromboxane $\mathrm{A}_{2}$ generation. Various platelet activators including thrombin and collagen produce a sufficiently brisk increase in the amount of free cytoplasmic calcium to cause directly the release reaction, bypassing the mediation of the arachidonic acid cascade. ${ }^{7}$ Calcium is released from the dense tubular system and influxes from the external medium, and this free calcium forms a complex with calmodulin; the calcium-calmodulin complex acts as a coenzyme in a series of platelet reactions. It initiates granule release, liberates arachidonic acid from the membrane phospholipids, making it available for conversion into thromboxane $\mathrm{A} 2$, and activates the actomyosin contractile system of filaments beneath the platelet membrane.

The third pathway entails the release of a lysolecithin compound called PAF (platelet activating factor) from the platelet membrane phospholipids and this seems to be able to activate platelets independently of thromboxane A2 generation and calcium release. ${ }^{8}$ The actual importance of PAF activation of platelets in man has not yet been fully determined.

The aggregating platelets align together into, initially, a rather loose reversible aggregate, but after the release reaction of the platelet granules a larger, interdigitating, irreversible plug is formed. Changes in the platelet membrane configuration now occur, entailing a "flip-flop" rearrangement of the membrane surface: the coagulant active negatively charged phospholipids, phosphatidylserine and phosphatidylinositol, which are concentrated on the inner half of the phospholipid layer, become exposed on the outer surface of the activated platelet. ${ }^{9}$

Platelets do not normally adhere to undamaged endothelial cells, and over the past decade it has been shown that vascular endothelium has an important role in the control of the haemostatic process. ${ }^{10}$ Endothelial cells synthesise prostacyclin from arachidonic acid, which, when released into the circulation, causes local vasodilation and is the most potent known natural inhibitor of platelet adhesion and aggregation. ${ }^{11}$ Prostacyclin probably does not circulate in biological quantities, but it is released locally when the vessel wall is stressed or injured, thus controlling any excessive platelet activation. When it binds to specific platelet membrane receptors it activates membrane bound adenylate cyclase, which produces increased levels of cyclic adenosine- $5^{\prime}$ monophosphate (cAMP). This inhibits platelet aggregation by inhibiting arachidonic acid metabolism and internal calcium flux. The vascular endothelial cell also synthesises and stores prostaglandin $\mathrm{E}_{1}$, factor VIII related antigen/von Willebrand factor, fibronectin, and a heparin like material, which also influences the control of platelet activation.

\section{Initial screening tests for a platelet defect}

The peripheral platelet count and the skin bleeding time are the first line basic laboratory tests of platelet function. If these two tests are within normal limits it is unlikely that a clinically important platelet defect is responsible for excessive clinical bleeding. Automated cell counters are now widely available, and these count platelets quickly and reliably. They also provide an assessment of mean platelet volume, total platelet crit, and a distribution graph of platelet size. The interpretation of these variables has recently been reviewed, ${ }^{12}$ but their clinical importance is doubtful. Additional information may be gained by inspecting a fresh blood film, which may show abnormalities of platelet size or morphology, and these can be of diagnostic importance.

There are several methods for performing a bleeding time test (Ivy, Duke, and template methods), all of which rely on the time taken for a standard skin incision, breaking small subcutaneous vessels, to stop bleeding. ${ }^{13}$ The reproducibility, however, is very variable and depends on several factors, including operator experience, skin temperature, venous pressure, direction, and length and depth of incision. The template technique, using a disposable spring loaded device (such as Simplate II, General Diagnostics), standardises at least the last three variables and should give reasonably comparable results for clinical use. It is important that each laboratory establishes a normal range for the exact method used, as a recent survey of British laboratories reported a great deal of variation in the normal range, even when a template technique was used. ${ }^{14}$ 
A prolonged bleeding time occurs when the platelet count falls below roughly $100 \times 10^{9} / 1$, and there is a progressive increase as the platelet count falls below this. ${ }^{15}$ If the platelet count is normal or raised a prolonged bleeding time suggests one of the many congenital or acquired qualitative disorders of platelet function. ${ }^{1617}$ Other non-platelet haemostatic disorders, which may prolong the bleeding time, are vascular abnormalities, particularly if they entail skin collagen defects, von Willebrand's disease, afibrinogenaemia, dysproteinemias, severe anaemia with a haematocrit less than $0 \cdot 20$, and conditions in which the vessel wall may produce increased amounts of prostacyclin like material-such as chronic renal failure.

If such screening procedures suggest a platelet functional disorder, or if there is a high degree of clinical suspicion despite a normal bleeding time, further tests should be planned in a systematic way. Drugs and certain dietary practices are the commonest causes of platelet dysfunction, and patients must refrain from taking drugs with known antiplatelet effects for seven to 10 days before blood sampling for more specific investigation of function. Table 2 lists the most important of these drugs and dietary food stuffs. ${ }^{18}$

\section{Platelet adhesion tests}

There are several variations on the basic technique developed by Baumgartner in 1963. ${ }^{19}$ These rely on

Table 2 Substances that commonly affect platelet function

\footnotetext{
1 Membrane stabilising agents:

$\alpha$-antagonists

$\beta$-blockers

Local anaesthetics (procaine)

Antihistamines

Tricyclic antidepressants

2 Agents that affect prostanoid synthesis: Aspirin

Non-steroid anti-inflammatory drugs

Corticosteroids

3 Antibiotics:

Penicillin

Cephalosporins

4 Agents that increase cyclic AMP activity

Dipyridamole

Aminophylline

Prostanoids

5 Others:

Heparin

Dextrans

Ethanol

Phenothiazine

Clofibrate

Garlic

Black tree fungus
}

whole blood passing over an everted segment of rabbit aorta, with a denuded endothelial surface in a continuous flow circuit at $37^{\circ} \mathrm{C}$. The extent of platelet adhesion is then assessed by light microscopy of the aortic surface and quantitation of adhesion can be measured if indium-radiolabelled platelets are used in the flow circuit. The shear rate over the vessel surface can be changed to mimic in vivo conditions in different sized blood vessels. Because this technique is expensive, time consuming, and technically difficult, however, it should be used only for specific research purposes.

The most widely adopted diagnostic adhesion test measures platelet retention after a single passage of whole blood through a glass bead column. Attempts to standardise this test have used glass beads of a constant size packed in a tube of fixed diameter and length (which are commercially available), with a steady infusion rate of non-anticoagulated whole blood directly from an arm vein, using an evacuated system. The percentage platelet retention in the column is calculated from a pre-column and post column infusion platelet count. Despite all these precautions the results are extremely variable, and decreased glass bead retention of platelets is not only found in platelet adhesion defects. It has subsequently been shown that retention also depends on plasma von Willebrand factor (factor VIII:vWF) activity, fibrinogen coating the glass bead surface, and on platelet aggregation, which is promoted by local release of ADP from haemolysed red cells in the column. Because of this lack of specificity, these tests are not widely used.

\section{Platelet aggregation}

Studies of platelet aggregation are mandatory in anyone suspected of having a platelet function defect, particularly in patients with a prolonged or borderline bleeding time. These tests are routinely performed on platelet rich plasma, although techniques using whole blood have recently been developed.

\section{TURBIDOMETRIC TECHNIQUE}

Platelet rich plasma is prepared from citrated whole blood (usually $1: 10,3 \cdot 13 \%$ trisodium citrate:blood) by centrifugation at about $180 \times g$ for 10 minutes. The standard method utilises an optical aggregometer and is based on the turbidometric method of Born. ${ }^{20}$ This works on the principle that when platelets are in a suspension aggregate the turbidity of the suspension falls, and light transmission through it increases proportionally. Most workers dilute the platelet rich plasma to a final platelet count of $200-300 \times 10^{9} / 1$ with autologous platelet poor plasma. It is important to standardise testing of aggregation, and ideally this 


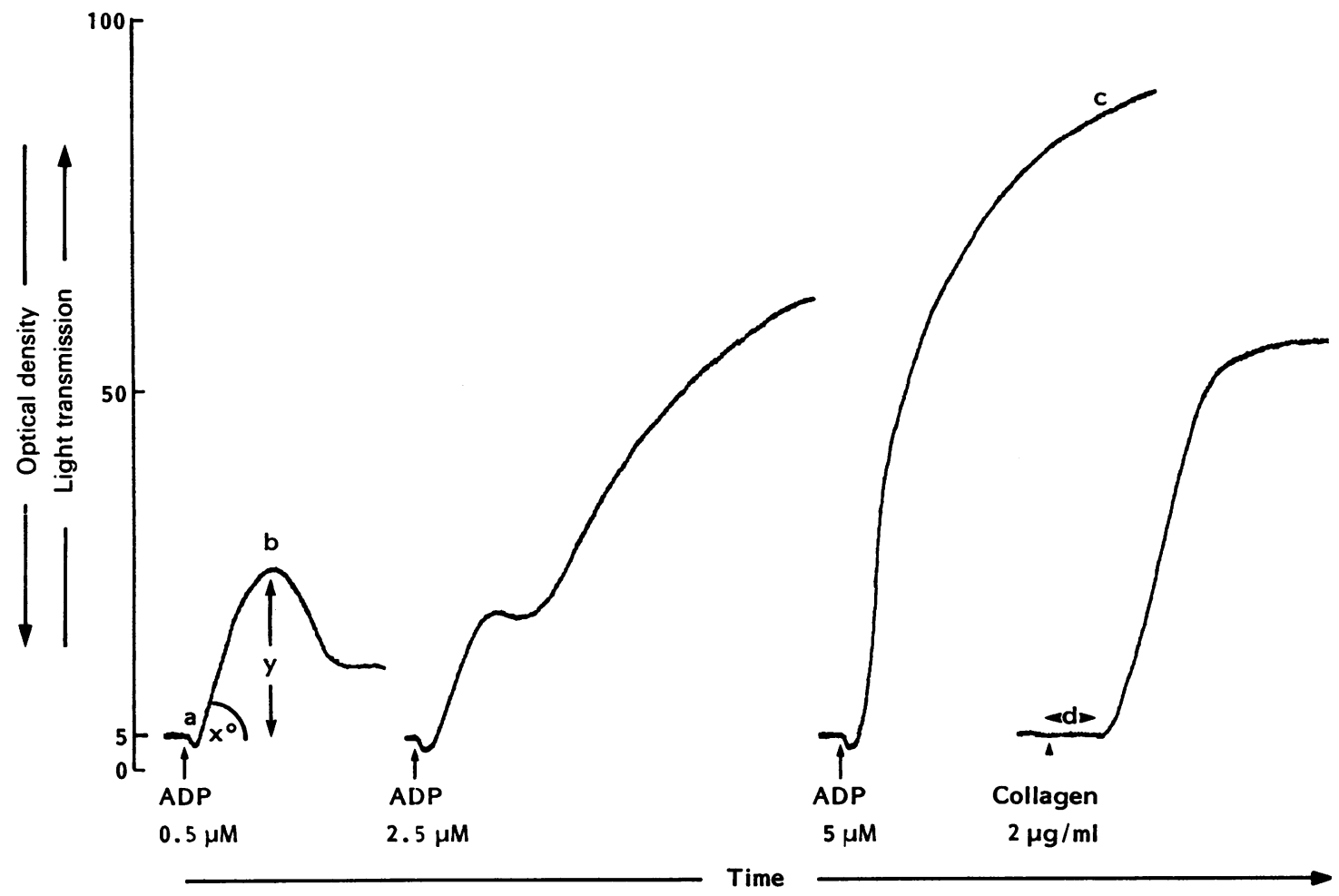

Fig. 2 Typical traces obtained during platelet rich plasma aggregation. $A D P=$ adenosine 5 -diphosphate; $a=$ shape change; $b=$ primary wave aggregation; $c=$ secondary wave aggregation; $x^{\circ}=$ angle of ascent of aggregation trace; $y=$ height of aggregation trace; $d=$ lag phase.

should be performed one to three hours after collection of the blood. Aliquots of the diluted suspension are placed in a glass cuvette in an aggregometer, positioned in a heating block kept at $37^{\circ} \mathrm{C}$, and continuously stirred by means of a small metal stir bar in each cuvette and a rotating magnet in the aggregometer beneath. The turbidity of the suspension is constantly measured by recording transmission of a light beam directed through it, and it is recorded as a change in voltage on a chart recorder. As platelets aggregate in response to an added agonist, light transmission increases progressively, producing an "aggregation trace" on the recorder. The machine is calibrated for each sample so that the platelet suspension to be studied reads $5 \%$-that is, minimum transmission-and the corresponding platelet poor plasma reads $95 \%$ (maximum transmission). Fig. 2 shows some typical traces obtained with some commonly used aggregating agents. Measurement of the aggregation response may be made by measuring the angle $(x)$ of the initial aggregation slope and the maximum height $(y)$ of the response after a standard time (usually three minutes). Satis- factory responses can be obtained with volumes of platelet rich plasma of at least $0.2 \mathrm{ml}$ in most modern aggregometers and with platelet counts as low as 100 $\times 10^{9} / 1$. In newborn infants, however, it may be impossible to obtain a sufficient volume of platelet rich plasma, and to avoid these problems a technique utilising radiolabelled monoclonal antibodies to specific surface membrane glycoproteins using capillary blood samples has been developed. ${ }^{21}$

There are numerous aggregating agents, or agonists, that will activate platelet aggregation (Table 3). For routine diagnostic screening virtually all of the clinically important aggregation defects will be detected by using various concentrations of ADP, collagen, and ristocetin. Confirmatory information may be obtained with additional agonists.

\section{$A D P$}

ADP binds to a specific platelet membrane receptor associated with the glycoprotein GPIIb/GPIIIa complex and extracellular calcium ions. Extracellular fibrinogen then binds in a reversible fashion to this activated membrane complex. After initial activation 
Table 3 Antagonists for platelet aggregation studies

Final concentrations usually used

A Agents routinely used in screening tests:

ADP

Collagen

*Ristocetin

B Other agents in routine use:

Adrenaline

Thrombin

Arachidonic acid

Endoperoxide analogues U44069 and U46619

Calcium ionophores A23187

C Agents used chiefly for research purposes.

Platelet activating factor

Serotonin

Antigen-antibody complexes

Platelet antibodies

Viruses, bacterial products

Particles, such as latex and absorbed $\mathrm{C} 3$ and fibrinogen

* Bovine factor VIII

*Concanavalin A

*Phytohaemagglutinins

*Agents causing platelet agglutination rather than true aggregation.

**This varies according to species and tissue source of collagen. The manufacturer's instructions should be followed.

the platelets undergo a change in shape from a disc shaped cell to a spherical form with protruding pseudopodia. This is reflected by a transient increase in turbidity of the platelet suspension and an initial small down turn of the aggregation trace (Fig. 2). After this change in shape initial reversible aggregate formation occurs, and bound fibrinogen aids cell to cell contact. Such primary wave aggregation, when stimulated by low concentrations of ADP - that is, $0.5-1.0 \mathrm{mM}-$ is reversible with subsequent disaggregation and freeing of fibrinogen from the GPIIb/GPIIIa complex (Fig. 2). Higher concentrations of ADP $(2.5-5.0 \mathrm{mM})$ cause an irreversible secondary wave of aggregation that is associated with $\alpha$ and dense granule release (Fig. 2). ADP specifically stimulates the release of arachidonic acid and thromboxane $\mathrm{A}_{2}$ generation, inhibits adenylate cyclase activity, and thus lowers cAMP activity. This emphasises the importance of performing dose responses to various final concentrations of ADP (from 0.5-10.0 $\mathrm{mM}$ ) to detect some minor aggregation defects.

The secondary irreversible wave of platelet aggregation in response to ADP is abnormal or absent in several important situations:

1 Patients who have been taking aspirin or other non-steroidal anti-inflammatory drugs (Table 2), which inhibit the enzyme cyclo-oxygenase and therefore block the platelet prostaglandin pathway, show this abnormality. This is the commonest cause of a platelet aggregation abnormality.

2 Recent or regular ingestion of large quantities of alcohol also impairs the secondary aggregation response.

3 Several congenital abnormalities, including cyclooxygenase deficiency, thromboxane synthetase
$0.5-10.0 \mu \mathrm{M}$

$1 \cdot 0-4.0 \mu \mathrm{g} / \mathrm{ml}^{* *}$

$0.5-1.2 \mathrm{mg} / \mathrm{ml}$

$1 \cdot 0-10 \cdot 0 \mu \mathrm{M}$

$0 \cdot 1-0 \cdot 5 \mu / \mathrm{ml}$

$1.0-2.0 \mu \mathrm{M}$

$2.5 \mu \mathrm{g} / \mathrm{ml}$

deficiency, a thromboxane $A_{2}$ receptor abnormality, or a deficiency of the platelet granular contents, or defective release, will cause abnormal ADP induced aggregation. The most common of these is storage pool disease in which $\alpha$ or dense body granules, or both, are deficient, or their release mechanism is abnormal. Mild forms of storage pool disease may, however, have normal aggregation responses and can only be diagnosed by electron microscopy and measurement of specific granule release products after platelet stimulation.

\section{Collagen}

The physiological importance of platelet activation by collagen is evident, as the damaged vascular endothelial surface exposes subendothelial collagen fibres, initiating in vivo platelet activation. Platelets probably respond specifically to type III collagen, and this reaction depends on its primary and quaternary structure being intact. The mechanism of collagen interaction with the platelet membrane remains unclear, and binding to a specific membrane receptor has not been confirmed.

The aggregation response to collagen in vitro is $\mathrm{N}$ preceded by a short "lag phase" (Fig. 2) lasting for about 10 to 60 seconds, whose duration is inversely proportional to the concentration of the reagent used or the responsiveness of the platelets under study. Measurement of the length of the lag phase in $\stackrel{\infty}{?}$ response to given doses of collagen is important in the assessment of platelet responses to this agonist. This phase is succeeded by a single wave of aggregation. The major pathway of platelet activation by collagen is via the platelet prostaglandin pathway, with formation of thromboxane $\mathbf{A}_{2}$ from membrane phos- 
pholipids and release of granular contents by the stimulated platelets. Higher doses of collagen (2 $\mu \mathrm{g} / \mathrm{ml}$ or more), however, cause a sudden increase in intraplatelet calcium concentrations, and this may be sufficient to bring about the release reaction without the mediation of the prostaglandin pathway. Collagen is therefore usually used in two final concentrations: $1 \mu \mathrm{g} / \mathrm{ml}$ and $4 \mu \mathrm{g} / \mathrm{ml}$. The response to the lower dose is more specific for abnormalities of the arachidonic acid cascade, thromboxane $A_{2}$ generation, and subsequent granule release.

\section{Ristocetin}

The antibiotic ristocetin interacts with factor VIII related antigen/von Willebrand factor and glycoprotein GPlb on the platelet surface, inducing platelet aggregation. ${ }^{22}$ This aggregation is really an agglutination of the cells. In platelet rich plasma ristocetin causes a primary and secondary wave (due to granule release) of aggregation, but even with metabolically inert formalin fixed platelets irreversible agglutination occurs-independently of thromboxane $\mathbf{A}_{2}$ generation and granule release. The only important assessment of aggregation induced by ristocetin is the angle of the initial slope and not the height of maximal response, or the secondary wave.

Aggregation of ristocetin should initially be studied with a final concentration of $1.2 \mathrm{mg} / \mathrm{ml}$. Concentrations above $1.5 \mathrm{mg} / \mathrm{ml}$ may cause non-specific platelet clumping due to an interaction with fibrinogen and thus mask platelet defects. Defective aggregation is observed in von Willebrand's disease and the Bernard Soulier syndrome, in which the membrane glycoprotein GPlb is deficient. Platelet rich plasma from mild and some moderate forms of von Willebrand's disease will, however, agglutinate normally to ristocetin. Occasionally, defects in ristocetin aggregation may not be associated with any pathological abnormality and have been described in certain racial groups such as negroes. Subtype $11 \mathrm{~b}$ of von Willebrand's disease has also been reported in which increased aggregation induced by ristocetin occurs due to lack of the high molecular weight oligomers of factor VIII related antigen in plasma. ${ }^{23}$ This subtype can be detected by performing a dose response curve with ristocetin concentrations from $0.5-1.2 \mathrm{mg} / \mathrm{ml}$. Normal platelet rich plasma does not aggregate with concentrations of ristocetin below $1.0 \mathrm{mg} / \mathrm{ml}$ reliably, whereas subtype $11 \mathrm{~b}$ shows increased responses. A more specific and quantitative estimation of factor VIII related antigen/von Willebrand factor activity is the ristocetin cofactor assay using washed fixed normal platelets: ${ }^{24}$ this must be performed on the plasma of any patient suspected of having a factor VIII complex defect.

Alternative agonists to ristocetin, which also bind to platelet membrane GPlb, include bovine fibrinogen and botrocetin. In Bernard Soulier disease aggregation with these reagents is also defective.

\section{OTHER AGGREGATING AGONISTS}

Various other reagents are available whose chief function is to define further abnormalities detected by the use of the above three agonists.

\section{Adrenaline}

Platelets have roughly $100 \alpha_{2}$ receptors on each cell surface, and on binding to these adrenaline can promote aggregation. It is a weak agonist, and its chief physiological role is probably to act synergistically with other stronger activators. Thus subthreshold doses of ADP or collagen, which are themselves insufficient to cause platelet aggregation, may do so in the presence of a small "priming" dose of adrenaline. ${ }^{25}$

The platelet response to adrenaline is unusual in that there is no shape change preceding aggregation, but thereafter the response resembles that to ADP. Fibrinogen receptors are exposed initially, and a primary and secondary wave follows. The secondary wave is impaired in similar circumstances for both agents. Adrenaline is usually used at final concentrations of $1-10 \mu \mathrm{m}$.

Some clinically normal subjects have been reported as having severely reduced platelet responsiveness to adrenaline. ${ }^{26}$ In vitro aggregation responses to collagen and vasopressin may also be impaired. These findings may reflect a defect in cytosolic calcium mobilisation.

It should be noted that for adrenaline, and probably for collagen too, if platelet rich plasma is allowed to stand at room temperature for some time it becomes serially more responsive to these agonists. ${ }^{27}$ This change occurs over the first 30 minutes and then declines over the next 30 minutes. Caution must therefore be exercised when comparing results from studies in which this variable has not been standardised. Ideally, for maximum reproducibility, platelet rich plasma platelet aggregation studies should be carried out between one and three hours after the blood has been collected.

\section{Thrombin}

Studies of platelet activation by thrombin must be performed on washed platelet suspensions, as fibrinogen present in platelet rich plasma will cause rapid formation of a fibrin clot when exposed to thrombin, with consequent masking of any platelet responses.

Thrombin is a very powerful activator of platelet aggregation. It causes an immediate and profound rise in intraplatelet calcium concentrations and can 
directly activate the release reaction, independently of thromboxane $A_{2}$ generation. In lower doses, however, the arachidonic acid pathway and release of granular contents do contribute to the resulting aggregation.

It more potently activates washed platelets suspended in buffer than those suspended in platelet rich plasma, probably because of the removal of natural thrombin inhibitors present in plasma-mainly, antithrombin III and $\alpha_{2}$-macroglobulin. The dose range usually used is a final concentration of $0 \cdot 1-0.5 \mathrm{u} / \mathrm{ml}$.

\section{Arachidonic acid and cyclic endoperoxide analogues}

Responses to low dose thrombin or collagen are the most sensitive tests of platelet prostaglandin activation, as they require all the components of the arachidonic acid cascade to be functional before a normal response occurs. If this pathway is impaired study of platelet responses to individual components of the pathway is helpful in determining the site of the defect.

Exogenous arachidonic acid (final concentration 1-2 $\mathrm{mM}$ ) induces thromboxane $A_{2}$ generation and granule release, even if there is a defect of agonist binding to the platelet membrane, or in phospolipase induced release of endogenous arachidonate, from the membrane phospholipids. If steps further along the pathway are impaired, however, such as the inhibition or absence of cyclo-oxygenase, added arachidonic acid will not produce a normal aggregation response.

The synthetic endoperoxide analogues (U,46619 and $U, 44069$ ) are believed to act as thromboxane $A_{2}$ mimetics, and, as they bypass cyclo-oxygenase activity, are useful in defining more distal defects. If an abnormal response to thromboxane $A_{2}$ is suspected transfer experiments may be conducted; using the supernatant of maximally stimulated normal platelets added to the platelet rich plasma of stirred patient's platelets (and vice versa) ${ }^{28}$ In this way the biological function of thromboxane $A_{2}$ as an inducer of aggregation (which has a half life of only about 45 seconds) can be tested.

\section{Calcium ionophores}

These agonists can bypass the arachidonic acid pathway completely by causing a direct increase in free cytoplasmic calcium ions. The most useful ionophore is A23187, which at concentrations between 2.5-10.0 $\mu \mathrm{M}$, will aggregate platelets. Higher concentrations cause direct platelet lysis.

\section{Platelet activating factor (PAF)}

This lysolecithin compound was initially identified in patients with acute anaphylaxis and other allergic phenomena and is present in basophils, mast cells, neutrophils, monocytes, and platelet membranes. It is released following exposure to activated complement 으 components or ionophores. It can cause platelet aggregation independently of thromboxane $\mathrm{A}_{2}$ for- $\stackrel{\mathcal{O}}{\stackrel{D}{+}}$ mation, and probably of calcium ion mobilisation as $\bar{O}$ well.

The importance of specific defects in PAF induced aggregation alone are not clearly defined, and $\Phi$ whether this constitutes an independent third pathway in human platelet activation remains unclear.

\section{WHOLE BLOOD AGGREGATION}

There are several theoretical disadvantages in the study of platelet function by the standard turbidometric method that uses platelet rich plasma. is These include sample preparation by centrifugation, which removes the other cellular elements and a proportion of the heavier platelets from the final sample. 오 Red and white cells may influence platelet aggre- $ᄋ$ gation, and the timing of sample preparation allows certain labile modulators of platelet function to lose $\grave{c}$ their biological activity in standard aggregation studies.

To circumvent these problems platelet aggregation has recently been studied by means of whole blood aggregation techniques. Two main systems are available; impedance aggregometry ${ }^{29}$ and a method using a whole blood platelet counter. ${ }^{30}$ The impedance technique measures the progressive increase of electrical impedance between two electrodes as platelets adhere and aggregate to these electrodes in stirred whole blood. The other method uses a platelet counter: after an aggregating agent has been added to stirred whole blood the number of single free platelets are counted in subsamples taken at regular intervals. The percentage fall in the free platelet count gives an assessment of platelet aggregation.

Both these methods have been used mainly for research investigations, and their diagnostic advantages over the standard turbidometric technique are probably limited. The impedance method is very slow, requires standardisation of the haematocrit, and often very high non-physiological concentrations of aggregating agonists. Both techniques, however, ? permit more reliable study of thrombopenic samples with platelet counts as low as $50 \times 10^{9} / 1$ and assess- N ment of certain antiplatelet drugs, such as dipyridamole, which only inhibits aggregation in whole blood, ${ }^{31}$ and prostacyclin which has a short ex vivo half life.

\section{Assessment of granular content and the release} reaction

Inside platelets are three main forms of storage granules: $\alpha$ granules, dense bodies, and lysosomes. Platelet 
ultrastructure can be studied by transmission electron microscopy and in particular, the various types of granules can be visualised and their morphological changes observed during aggregation and granule release. Electron microscopy, in particular, is useful for diagnosing storage pool disease, grey platelet syndrome, and associated disorders of mixed organelle deficiency in which absent or decreased numbers of granules can be assessed.

\section{DENSE BODIES}

The dense bodies are the storage site for the nonmetabolic pool of adenine nucleotides, serotonin, and calcium. Nucleotides and serotonin can be readily measured and their release after platelet stimulation assessed. Methods for measuring intraplatelet calcium concentrations and flux have recently become available, but it is impossible at present to distinguish between dense body calcium release and other calcium fluxes following platelet activation.

\section{Adenine nucleotides}

The most commonly used diagnostic assay of adenine nucleotides uses a firefly bioluminescence technique. ${ }^{32}$ The measurement of the ATP content of a sample depends on the reaction:

Luciferin $+\mathrm{ATP}+\mathrm{O}_{2} \underset{\mathrm{Mg}^{+\rightarrow}}{\stackrel{\text { lucifere }}{\longrightarrow}} \mathrm{AMP}+\mathrm{PP}+\mathrm{CO}_{2}+$ oxyluciferin + ligh

High purity luciferase is now available, giving a directly proportional light output to the ATP concentration, and both end point and kinetic determinations can be made with a luminometer. This system measures ATP only; the ADP content of a sample can be assayed after converting all the ADP to ATP by the following reaction:

ADP + phosphoenolpyruvate $\stackrel{\text { PK. } \mathbf{M g}++}{\mathbf{K}+}$ ATP + pyruvate

Studies are usually performed on washed platelets to quantify total nucleotides and then after maximal stimulation with various suitable agonists, such as collagen and thrombin, on the supernatant of the platelet suspension to quantify release. ${ }^{33}$ The total nucleotide concentrations of normal human platelets in our laboratory are: ATP 5.4 (SD 0.3$) \mu \mathrm{M} / 10^{11}$ and ADP $3 \cdot 1(0.4) \mu \mathrm{M} / 10^{11}$ platelets, with 20 to $30 \%$ of ATP and 60 to $80 \%$ of ADP being released extracellularly after maximal stimulation with $20 \mu \mathrm{g} / \mathrm{ml}$ of collagen. This technique is very useful for the diagnosis of hereditary and acquired defects of dense granule contents and release.

An aggregometer system, incorporating a luminometer using the luciferase system, has recently been introduced, but the nucleotide measurements are only semiquantitative, and minor abnormalities may be missed. Alternative assay systems using high pressure liquid chromatography are very sensitive but are not widely available for diagnostic use.

\section{Serotonin release}

On incubation, radiolabelled ${ }^{14} \mathrm{C}$-serotonin is taken up by platelets and equilibrates with endogenous dense granule serotonin. The labelled platelets can then be stimulated and the released radiolabelled ${ }^{14} \mathrm{C}$-serotonin measured in the supernatant at various time intervals. ${ }^{34}$ To prevent reuptake of released serotonin imipramine must be added to the test system. As the absolute amount of serotonin in normal platelets is rather variable a normal laboratory range must be established for each agonist.

\section{Alpha granules}

The $\alpha$ granules contain several specific proteins and coagulation factors, and after platelet activation these granules release their contents. Release of $\alpha$ granules requires less powerful stimulation of the cells than release of dense granules, so that there can be evidence of $\alpha$ degranulation with the dense granules remaining intact. These platelets can still be further stimulated and go on to release their dense granular contents. Three storage proteins - namely, platelet factor 4 (PF4), $\beta$-thromboglobulin ( $\beta$-TG), and platelet derived growth factor (PDGF or mitogenic factor) are platelet specific and are therefore useful in assessing minor degrees of platelet activation.

\section{PLATELET FACTOR 4 AND $\beta$-THROMBOGLOBULIN}

Platelet factor 4 is a protein with a molecular weight of 35000 , comprising four identical subunits each with a molecular weight of about 8000 . It is released bound to a proteoglycan carrier molecule. Vascular endothelial cells synthesise and release heparin sulphate, which catalyses the neutralisation of thrombin by antithrombin III when the haemostatic system is locally activated. PF4 readily binds to heparin and similar substances on the endothelial surface and can therefore delay thrombin neutralisation after platelet release. In vitro it is also chemotactic for monocytes and neutrophils, inhibits the activity of skin and leucocyte collagenases, and has various other reported activities of uncertain biological importance. It has a half life of roughly two minutes, the rapid clearance possibly reflecting its binding to endothelial cells: PF4 is not excreted to any appreciable degree by the kidney. The normal range in plasma-as measured by radioimmunoassay - is $2-20 \mathrm{ng} / \mathrm{ml}$, whereas the unstimulated platelet content is about $18 \mathrm{mg} / 10^{9}$ platelets. Plasma concentrations are falsely increased during treatment with heparin, probably due to displacement of bound PF4 from endothelial cell binding sites.

$\beta$-TG is also a tetrameric protein, with a molecular 
weight of about 36000 . It is released from platelets as "low affinity PF4." Removal of some terminal amino acids of the subunits in the circulation releases $\beta$-TG. $\beta$-TG also binds to vascular endothelium and has been reported to inhibit production of prostacyclin. The half life of $\beta-\mathrm{TG}$, however, is considerably longer than that of PF4 - about 100 minutes - with an initial phase of excretion of nine minutes. $\beta$-TG has a wide normal range in plasma: $7-50 \mathrm{ng} / \mathrm{ml}$, with an average platelet content of $17.7 \mathrm{mg} / 10^{9}$ platelets. As $\beta$-TG is catabolised by the kidney, impaired renal function will produce increased plasma concentrations, which are not indicative of excessive in vivo platelet activation.

Several commercial kits are available for radioimmunoassay of these two proteins in plasma, but the techniques entailed pose some difficulties if the results are to be reproducible. ${ }^{35}$ Of particular importance is the method of sample collection, as any activation of platelets at this time induces secretion and falsely raises the final assay result. The sample should be taken without venous stasis into a chilled syringe after discarding the first $5 \mathrm{ml}$ of blood collected. It should then be transferred immediately to a plastic or siliconised tube on melting ice, containing a mixture of inhibitors to minimise in vitro release. The mixture commonly includes edetic acid, theophylline, and sometimes also an inhibitory prostaglandin, $\mathrm{PGE}_{1}$. The sample can briefly be kept on ice, but as soon as possible must be cold spun at $4^{\circ} \mathrm{C}$ for 10 minutes at $3000 \mathrm{~g}$ and the top $1 \mathrm{ml}$ of supernatant stored at $-50^{\circ} \mathrm{C}$ until assay.

When renal failure occurs PF4 is the more reliable indicator of in vivo release, whereas during treatment with heparin $\beta$-TG will give more reliable results. In general, however, the best measure of in vivo (as opposed to in vitro) release can be obtained from simultaneous assay of both proteins. Because of the more rapid clearance of PF4 from the circulation, and because during release the two are released in parallel in virtually similar amounts, genuine in vivo granule release is reflected by a higher $\beta$-TG than PF4 value. If in vitro activation has occurred-for example, during sample collection-the plasma PF4 value will approach that of $\beta$-TG, and this information is useful for detecting artefactual increases in these proteins. Since these assays have become routinely available, measurements have been carried out in plasma from patients with a wide variety of prothrombotic conditions in an attempt to correlate excessive in vivo release with a hypercoagulable state.

Although plasma assays of PF4 and $\beta$-TG are usually used to detect hyperaggregable states, they are also useful for detecting $\alpha$-granule deficiency states, such as the hereditary defect of the grey platelet syndrome. After platelet stimulation with various ago-
Yardumian, Mackie, Machin

nists their release can be measured in the platelet supernatant.

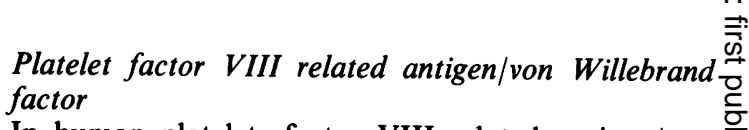
In human platelets factor VIII related antigen/von 흥 Willebrand factor is localised to the $\alpha$ granules, ${ }^{36}$ and $\overrightarrow{\widetilde{D}}$ after release binds preferentially to the platelet membrane. Recent evidence suggests that platelet factor $\%$ VIII related antigen/von Willebrand factor may con- $\overrightarrow{0}$ tain a higher proportion of high molecular weight multimers of factor VIII related antigen than plasma $\vec{\omega}$ factor VIII related antigen/von Willebrand factor. Analysis of platelet VIII:vWF is useful in the diagno- ? sis of certain variants of von Willebrand's disease.

\section{Platelet derived growth factor (PDGF)}

Platelet derived growth factor is a granule protein 음 with extremely interesting properties. It has two poly- peptide chains linked by disulphide bonds, with a molecular weight of 33000 . It binds to a specific $₹$ receptor on the surface of cells that are mitogenically $\vec{\bullet}$ responsive, including human arterial smooth muscle $\stackrel{\circ}{\circ}$ cells and fibroblasts. It is also chemotactic for monocytes and polymorphs. PDGF is itself, however, unable to promote growth of these cells, but it is important in preparing cells for the action of other growth promoters, possibly somatomedin. Ross et al first proposed that by mediating a cellular proliferation response PDGF may contribute to the observed initial intimal thickening that is such an important feature of atheromatous lesions. ${ }^{37}$ Assays for this protein are not yet routinely available, but following the amino acid sequencing of the molecule they may soon be.

\section{Further investigation of the platelet prostaglandin pathway}

If platelet aggregation studies suggest an abnormality in the arachidonic acid cascade more specific quantitation of these metabolites is now possible. Thromboxane $\mathrm{B}_{2}\left(\mathrm{TXB}_{2}\right)$, the nonenzymatic degradation $N$ product of thromboxane $A_{2}$, is readily quantified by radioimmunoassay. ${ }^{38}$ Thus $\mathrm{TXB}_{2}$ assays in plasma give an indication of in vivo platelet prostaglandin activation, whereas after specific platelet activation they quantitate the degree of arachidonic acid release $\stackrel{\oplus}{\mathscr{D}}$ and metabolism. Similar quantitation of thromboxane $A_{2}$ generation can be assessed by the assay of malondialdehyde, using the thiobarbituric acid method. ${ }^{39}$ The other intermediates of the pathway can be quantitated by incubating platelets with radiolabelled arachidonic acid and separating the 
products formed after platelet activation by thin layer chromatography. ${ }^{40}$ Alternatively, specific radioimmunoassays for the primary prostaglandin and leucotriene products can be developed.

\section{Platelet coagulant activity}

After platelet activation and completion of the release mechanism various coagulation supporting interactions occur at the platelet surface membrane. The biochemical importance of the flip-flop rearrangement of the negatively charged membrane phospholipids and their interaction with factor $\mathrm{X}$ and prothrombin have been well described elsewhere. ${ }^{9}$ Routine assessment of these interactions relies on the prothrombin consumption index. This test measures residual clotting activity in serum after clotting of whole blood by the time taken for prothombin consumption. ${ }^{41}$ The ability of serum is compared with that of citrated platelet poor plasma and the result expressed as the prothrombin time of serum divided by that of plasma (normal range $<20 \%$ ). Obviously, abnormalities will occur if a coagulation factor deficiency, thrombocytopenia, or any other platelet function defect is present. It remains a useful screening test for platelet dysfunction, however, especially if more sophisticated methodology is not available. In some subjects with a bleeding tendency this remains the only detectable haemostatic defect. ${ }^{42}$

PF3 activity was originally described as the contribution of platelets to the activation of prothrombin by factor Xa. This test has now virtually disappeared as reduced activity measured in this way is usually secondary to well defined platelet defects. The original assay is not only dependent on the surface exposure of procoagulant phospholipids, but is also influenced by platelet aggregation and fibrinogen binding to the platelet membrane. More specific assays measuring the catalysis of factor $\mathrm{X}$ activation and the conversion of prothrombin into thrombin on the activated platelet surface completely independently of platelet aggregation and granule release are now available but are usually used during research. ${ }^{43}$

\section{Platelet calcium changes}

As calcium is probably the single most important mediator in platelet activation it is important to quantify changing concentrations of this ion in response to varying degrees of platelet stimulation. A method has recently become available in which free intracytosolic calcium concentrations can readily be quantified $^{44}$; at present it is chiefly a research tool, but it may become more widely used.

Quin-2 is a fluorescent tetracarboxylic acid, which chelates calcium preferentially over other metal ions, and on so doing, its fluorescence increases by a factor of $\times 5-6$. It can be induced to enter cells by esterification (a hydroxymethyl ester), and on entering the cells it is hydrolysed back to the free acid that is "trapped" inside the cell. ${ }^{45}$ When the platelets are stimulated there is a very rapid rise in free cytoplasmic calcium, which can be monitored by the rise in fluorescent signal emitted at the appropriate wavelength. This is measured in a modified fluorimeter, which keeps the platelet suspension stirred and at $37^{\circ} \mathrm{C}$. Thus in response to thrombin, for example, the free calcium rises from a resting concentration of about $100 \mathrm{mM}$. The technique does not distinguish between calcium released from intracellular stores and that influxing from the surrounding medium, but it has been suggested that most is extracellular as the rise is considerably blunted if the reactions are carried out in a calcium free media. Some response does remain, however, so that undoubtedly some calcium is rapidly released from the dense tubular system and dense granules in response to cell activation.

It may be useful to assess otherwise unclassifiable platelet defects in such a system. ${ }^{28}$ With the increasing clinical use and development of calcium channel blocking drugs such as verapamil and nifedipine, investigation of calcium changes will become more important.

\section{Conclusions}

We have outlined the basic understanding and interpretation of the currently available methodology for investigating patients with presumed platelet defects and a haemorrhagic syndrome. Most of these tests should be available in the larger haemostasis units; and procedures that are mainly used in research have only been briefly mentioned or excluded.

\section{References}

${ }^{1}$ Sixma JJ, Wester J. The haemostatic plug. Semin Haematol 1977;3:265-99.

${ }^{2}$ Vermylen J, Badenhorst PN, Deckmyn H, Arnout J. Normal mechanisms of platelet function. Clinics Haematol 1983;12:107-52.

${ }^{3}$ Zimmerman TS, Ruggeri ZM, Fulcher CA Factor VIII/von Willebrand Factor. In: Brown EB, ed. Progress in haematology. Vol XIII. New York: Grune and Stratton, 1983:279-310.

${ }^{4}$ Born GV, Bergguist D, Arfers KE. Evidence for inhibition of platelet activation in blood by a drug effect on erythrocytes. Nature 1976;259:233-5.

${ }^{5}$ Smith JB. In: Bloom AL, Thomas DP, eds. Haemostasis and thrombosis. Edinburgh: Churchill Livingstone 1981:61-72.

${ }^{6}$ Marcus AJ. Platelet arachidonic acid metabolism. In: Harker LA, Zimmerman TS, eds. Measurements of platelet function. Edinburgh: Churchill Livingstone 1981:126-43.

${ }^{7}$ White GC. Calcium-dependent proteins in platelets. Biochim Biophys Acta 1980;631:130-9.

${ }^{8}$ Chignard M, Le Couedic JP, Vorgaftig BB, Benueniste J. Plateletactivating factor (PAF-acether) secretion from platelets: effect of aggregating agents. Br J Haematol 1980;46:455-64. 
${ }^{9}$ Zwaal RFA, Hemker HC. Blood cell membranes and haemostasis Haemostasis 1982;11:12-39.

${ }^{10}$ Thorgeirsson G, Robertson AL. The vascular endotheliumpathobiologic significance. Am J Pathol 1978;93:803-48.

${ }^{11}$ Whittle BJR, Moncada S. Pharmacological interactions between prostacyclin and thromboxanes. Br Med Bull 1983;39:232-8.

${ }^{12}$ Corash L. Platelet sizing: techniques; biological significance and clinical applications Current Top Haematol 1983;4:99-123.

${ }^{13}$ Nieuwenhuis HK, Sixma JJ. Bleeding time measurements. In: Harker LA, Zimmerman TS, eds. Measurement of platelet function. Edinburgh: Churchill Livingstone, 1983:26-45.

${ }^{14}$ Poller L, Thompson JM, Tanenson JA. The bleeding time: current practice in the UK. Clin Lab Haematol 1984;6:369-73.

${ }^{15}$ Marker LA, Slichter SJ. The bleeding time as a screening test for evaluation of platelet function. New Engl $J$ Med 1972;287:999-1005.

${ }^{16}$ Hardisty RM. Hereditary disorders of platelet function. Clin Haematol 1983;12:153-74.

${ }^{17}$ Raio AK, Walsh PN. Acquired qualitative platelet disorders. Clin Haematol 1983;12:201-38.

${ }^{18}$ Packham MA, Mustard JF. Clinical pharmacology of platelets. Blood 1977;50:555-67.

${ }^{19}$ Turitto VT, Baumgartner HR. Platelet adhesion. In: Harker LA, Zimmerman TS, eds. Measurement of platelet function. Edinburgh: Churchill Livingstone, 1983:46-63.

${ }^{20}$ Born GVR. Quantitative investigations into the aggregation of blood platelets. J Physiol 1962;162:67-71.

${ }^{21}$ Montgomery RR, Kunicki TJ, Taves C, Pidard D, Corcoran M. Diagnosis of Bernard-Soulier syndrome and Glanzmann's thrombasthenia with a monoclonal assay in whole blood. J Clin Invest 1983;71:385-9.

${ }^{22}$ Howard MA, Firkin BG. Ristocetin-a new tool in the investigation of platelet aggregation. Thrombosis Et Diathesis Haemorrhagica 1971;26:362-9.

${ }^{23}$ Zimmerman TS, Ruggeri AN. Von Willebrand's disease. Clin Haematol 1983;12:175-200.

${ }^{24}$ Weiss HJ, Moyer LW, Rickles FR, Varma A, Rogers J. Quantitative assay of a plasma factor deficient in von Willebrand's disease that is necessary for platelet aggregation. J Clin Invest 1973;52:2708-10.

${ }^{25}$ Mills DCB, Roberts GCK. Effect of adrenaline on human blood platelets. J Physiol 1967;193:443-53.

${ }^{26}$ Scrutton MC, Clare KA, Hutton RA, Bruckdorfer KR. Depressed responsiveness to adrenaline in platelets from apparently normal human donors-A familial trait. Br J Haematol 1981;49:303-14.

${ }^{27}$ Rossi EC, Louis G. A time-dependent increase in the responsiveness of platelet-rich plasma to epinephrine. J Lab Clin Med 1975;43:300-16.

${ }^{28}$ Hardisty RM, Machin SJ, Nokes TJC, Rink TJ, Smith SW. A new type of congenital defect of platelet secretion: impaired sensitivity of platelets to free cytoplasmic calcium ion. $\mathrm{Br} J$ Haematol 1983;53:543-57.

${ }^{29}$ Mackie IJ, Jones R, Machin SJ. Platelet impedance aggregation in whole blood and its inhibition by antiplatelet drugs. J Clin Pathol 1984;37:874-8.
${ }^{30}$ Lumley P, Humphrey PPA. A method for quantitating platelet aggregation and analysing drug receptor interactions on platelets in whole blood in vitro. $J$ Pharmacol Methods 1981;6:153-66.

${ }^{31}$ Gresele P, Zaja C, Deckmyn H, Arnaut J, Vermylen J, Verstraete $M$. Dipyridamole inhibits platelet aggregation in whole blood.ర Thromb Haemost 1983;50:852-6.

${ }^{32}$ David JL, Herion F. Assay of platelet ATP and ADP by the $\frac{\bar{\sigma}}{\bar{\omega}}$ luciferase method. Adv Exp Med Biol 1972;34:341-9.

${ }^{33}$ Summerfield GP, Keenan JP, Brodie NJ, Bellingham AJ. Bio- ${ }_{\overparen{D}}^{\circ}$ luminescent assay of adenine nucleotides: rapid analysis of ATP and ADP in red cells and platelets using the LKB luminometer. Clin Lab Haematol 1981;3:257-71.

${ }^{34}$ Murer EH. Use of pre-absorbed serotonin for measuring the release reaction. In: Day $H J$, Halmsen $H$, Zucker $M B$, eds. $\vec{\omega}$ Platelet function testing. US Department of Health, Education and Welfare, 1978:142-9.

${ }^{35}$ Ludlam CA, Moore S, Balton AE, Pepper DS, Cash JD. The? release of a human platelet specific probe measured by a radioimmunoassay. Thromb Res 1975;6:543-8.

${ }^{36}$ Zicker HB, Broekman MJ, Kaplan KL. Factor VIII related antigen in human platelets. $J$ Lab Clin Med 1979;94:675-82.

${ }^{37}$ Ross R, Glomset J, Kariya B, Harker L. A platelet-dependent serum factor that stimulates the proliferation of arterial smooth $\mathrm{O}$ muscle cells in vitro. Proc Nat Acad Sci USA 1974;71:1207-10. -

${ }^{38}$ Pang SS, Levine L. Prostaglandin biosynthesis and metabolism measured by radioimmunoassay. Prostaglandins 1977;3:41-76.

${ }^{39}$ Feinstein MB, Becker EL, Fraser C. Thrombin, collagen and A23187 stimulated endogenous platelet arachidonate metabo- $\overrightarrow{0}$ lism, differential inhibition by $\mathrm{PGE}_{1}$, local anaesthetics and aserine-protease inhibitor. Prostaglandins 1977;14:1075-93.

${ }^{40}$ Defreyn G, Machin SJ, Carreras LO, Dauden VM, Chamone DAF, Vermylen J. Familial bleeding tendency with partial platelet thromboxane synthetase deficiency. Br J Haematol 1981; 49:29-42.

${ }^{41}$ Parry DH, Giddings JC, Bloom AL. Familial haemostatic defect associated with reduced prothrombin consumption. $\mathrm{Br} J \mathrm{Hae}-\stackrel{\mathbb{Q}}{\varrho}$ matol 1980;44:323-34.

${ }^{42}$ Giddings JC, Peake IR. Laboratory support in the diagnosis of coagulation disorders. Clin Haematol 1985;14:571-95.

${ }^{43}$ Rosing J, van Rijn JLML, Bevers EM, von Dieijen G, Comfurius $P$, Zwaal RFA. The role of activated human platelets in pro-O thrombin and factor X activation. Blood 1985;65:319-32.

${ }^{44}$ Rink TJ, Smith SW, Tsien RY. Cytoplasmic free calcium in human platelets: calcium thresholds and calcium independent 0 activation for shape change and secretion. Febs Lett 3 1982;148:21-6.

${ }^{45}$ Tsien TY, Pozzan T, Rink TJ. Calcium haemostasis in intact lym- $\varrho$ phocytes; cytoplasmic free calcium monitored with a new intracellularly trapped fluorescent indicator. $J$ Cell Biolo 1982;94:325-34.

Requests for reprints to: Dr SJ Machin, Department of Haematology, Middlesex Hospital, London W1, England $\sigma$ 